\title{
Preparing for (valenced) action: The role of differential effort in the orthogonalized go/no-go task
}

\author{
HANNE SCHEVERNELS, KLAAS BOMBEKE, RUTH M. KREBS, AND C. NICO BOEHLER \\ Department of Experimental Psychology, Ghent University, Ghent, Belgium
}

\begin{abstract}
Associating reward to task performance has been shown to benefit scores of cognitive functions. Importantly, this typically entails associating reward to the execution of a response, hence intertwining action-related processes with motivational ones. However, recently, preparatory action requirements (go/no-go) and outcome valence (reward/ punishment) were elegantly separated using a cued orthogonalized go/no-go task. Functional magnetic resonance imaging results from this task showed that typical areas of the "reward network," like the dopaminergic midbrain and the striatum, predominantly encode action rather than valence, displaying enhanced activity when preparing for action (go) compared to inaction (no-go). In the current study, we used ERPs to probe for differences in preparatory state related to cognitive effort in this task, which has similarly been linked to reward-network activity. Importantly, the contingent negative variation, which is linked to effortful cognitive preparation processes during cue-target intervals, was clearly observed in go trials but not in no-go trials. Moreover, target-locked ERP results (N1 and P3) suggested that attention to the target was enhanced when an action had to be performed (go trials), and typical inhibition-related ERP components were not observed in no-go trials, suggesting a lack of active response inhibition. Finally, feedback-related P3 results could suggest that correct feedback was valued more in motivated go trials, again implying that more effort was required to correctly perform the task. Together, these results indicate that the anticipation of action compared to inaction simultaneously entails differences in mental effort, highlighting the need for further dissociation of these concepts.
\end{abstract}

Descriptors: Effort, Preparation, Motivation, Event-related potentials, Contingent negative variation

Everyday behavior requires flexible control that is sensitive to changes in the environment. When the environment or context suggests the availability of reward or punishment, people strive for minimization of punishment and the maximization of reward. As such, it has been shown that the prospect of reward enhances performance in a wide range of behavioral tasks (Adcock, Thangavel, Whitfield-Gabrieli, Knutson, \& Gabrieli, 2006; Beck, Locke, Savine, Jimura, \& Braver, 2010; Etzel, Cole, Zacks, Kay, \& Braver, 2015; Locke \& Braver, 2008; Padmala \& Pessoa, 2011). However, in these tasks usually a predefined motor response has to be executed in order to receive a reward, thereby coupling reward to action execution (and the respective anticipation thereof). Hence, these studies cannot distinguish valence (i.e., reward vs. punishment) and action effects (i.e., action execution vs. inhibition) and their underlying neural substrates. This is of particular importance considering that, in Pavlovian control, valence and action are inherently linked since stimuli that are appetitive (either in nature or via classical conditioning) automatically invigorate approach behavior

This research was supported by the Ghent University Multidisciplinary Research Platform "The integrative neuroscience of behavioral control" and the Flemish Research Foundation (FWO; 3G022513 and FWO11/ $\mathrm{PDO} / 016)$.

Address correspondence to: Hanne Schevernels, Dept. of Experimental Psychology, Ghent University, Henri Dunantlaan 2, 9000 Ghent, Belgium. E-mail: h.schevernels@gmail.com or action and aversive stimuli promote withdrawal or inhibition. This relation between reward and the execution of a motor response (go) and between punishment and the inhibition of motor actions (no-go) has also been suggested in previous studies investigating learning and decision making (Chiu, Cools, \& Aron, 2014; Frank, Seeberger, \& O'Reilly, 2004; Freeman, Alvernaz, Tonnesen, Linderman, \& Aron, 2015; Gray \& McNaughton, 2000).

One group of studies (Cavanagh, Eisenberg, Guitart-Masip, Huys, \& Frank, 2013; Guitart-Masip et al., 2011; Guitart-Masip, Chowdhury et al., 2012; Guitart-Masip, Huys et al., 2012; Richter et al., 2014) that has elegantly investigated the effects of action requirements and reward value and their interaction has crossed both factors in a cue-based paradigm (the so-called orthogonalized go/no-go task). This led to four conditions indicated by different cues: go to win, go to avoid losing, no-go to win, and no-go to avoid losing, which were used to compare differential anticipatory neural activity related to action and valence (Guitart-Masip et al., 2011; Guitart-Masip, Chowdhury et al., 2012). The behavioral data favored an inherent association between reward and go indicated by an interaction between action and valence with increased accuracy in go trials when subjects anticipated monetary gains compared to losses (and similar accuracy levels in win and avoid-losing trials in the no-go condition). Nevertheless, fMRI results mostly demonstrated a main effect of action, in particular in parts of the typical "reward network" including the substantia nigra/ventral 
tegmental area (SN/VTA) and striatum that were found to predominantly encode action anticipation irrespective of valence (GuitartMasip et al., 2011; Guitart-Masip, Chowdhury et al., 2012).

However, recent evidence shows that the same neural system is also involved in effort-based management of neural resources. For example, Boehler et al. (2011) found that, even in the absence of reward or any other type of direct extrinsic motivator, the dopaminergic midbrain is more active when subjects prepare for a cognitively demanding task compared to a less demanding task. Moreover, it has been found that the dopaminergic midbrain regions and important dopaminergic target areas like the anterior cingulate cortex (ACC) and striatum are not only activated during the anticipation of reward but also during the anticipation of effort in a perceptual task (Krebs, Boehler, Roberts, Song, \& Woldorff, 2012), a cognitive task (Vassena et al., 2014), and a physical instrumental task (Kurniawan, Guitart-Masip, Dayan, \& Dolan, 2013). Hence, these results suggest that the dopaminergic midbrain is not only involved in the processing of reward but also in the control of cognitive processing resources (see also Nieoullon, 2002; Salamone, Correa, Mingote, \& Weber, 2005). As such, it is important to note that the action manipulation as described in the studies of Guitart-Masip and colleagues seems to simultaneously entail differences in mental effort. More specifically, in go trials, mental preparation is likely pronounced since subjects have to attend to the upcoming target in order to perform the task correctly (and quickly), which is not the case for no-go trials. Hence, activity enhancements of the striatum and dopaminergic midbrain during action-related preparation can alternatively or additionally represent changes in preparatory state.

In order to probe whether different levels of task preparation might have contributed to the dominating effect of action anticipation by Guitart-Masip's group, in the current study we recorded EEG activity while participants performed the orthogonalized go/ no-go task of Guitart-Masip et al. (2011) and Guitart-Masip, Chowdhury et al. (2012). To further test whether there is any preparatory effort during anticipation of no-go trials, we also included cued catch trials in which participants knew that no target would be presented (see also Grent-'t-Jong \& Woldorff, 2007). Moreover, two other baseline conditions were included, that is, neutral go and neutral no-go, in which trials were never rewarded or punished.

One event-related component that is particularly interesting with regard to the goal of the current study is the contingent negative variation $(\mathrm{CNV})$ since it is supposed to reflect changes in preparatory activity (Walter, Cooper, Aldridge, McCallum, \& Winter, 1964). The CNV is a frontocentral negative-going component observed between a warning and imperative stimulus that starts to appear around $1 \mathrm{~s}$ preceding target presentation and is supposed to reflect processes related to anticipatory attention, motor preparation, and arousal (Birbaumer, Elbert, Canavan, \& Rockstroh, 1990; Connor \& Lang, 1969; Tecce, 1972; van Boxtel \& Brunia, 1994). In a number of studies, the CNV was investigated using a cued go/ no-go paradigm, and they found that the CNV is larger when anticipating go trials compared to no-go trials, suggesting enhanced preparatory cortical activity (e.g., Filipović, Jahanshahi, \& Rothwell, 2001; Funderud et al., 2012; Rosahl \& Knight, 1995). Moreover, the $\mathrm{CNV}$ has been shown to be sensitive to the anticipation of different levels of task demands or cognitive effort (Ansari \& Derakshan, 2011; McEvoy, Smith, \& Gevins, 1998; Schevernels, Krebs, Santens, Woldorff, \& Boehler, 2014; but see Vuillier, Whitebread, \& Szucs, 2015), which may again largely reflect differences in preparatory state or the amount of resources allocated to a cognitive process (see also Rösler, Heil, \& Röder, 1997).
Therefore, if different levels of task preparation are involved, we would expect larger $\mathrm{CNV}$ amplitudes in anticipated go trials than in no-go trials (as suggested by previous studies). Since the CNV has been shown to be affected by extrinsic motivation (Hughes, Mathan, \& Yeung, 2012; Pierson, Ragot, Ripoche, \& Lesevre, 1987; Schevernels et al., 2014; van den Berg, Krebs, Lorist, \& Woldorff, 2014; but see Goldstein et al., 2006; Sobotka, Davidson, \& Senulis, 1992), we also expected a larger CNV amplitude in go to win and go to avoid-losing trials (which may or may not differ from each other) compared to go neutral trials. We also investigated the cue-evoked parietal P3 (which largely precedes the CNV) since this component has been shown to be affected by reward anticipation, suggesting its role in motivated attention (Goldstein et al., 2006, 2008; Hughes et al., 2012; Kohls et al., 2011; Schevernels et al., 2014).

The level of preparation should also affect processing of the subsequent target, and thus we also investigated target-evoked ERPs related to early visual processing and attention. Moreover, it is unclear how much active response inhibition precued no-go trials require (since the tendency to initiate a motor response is preempted by the cue) in which case one should not observe typical inhibition-related ERP effects, like modulations of the frontal target-evoked N2 and P3 in no-go trials (Bokura, Yamaguchi, \& Kobayashi, 2001; Bruin, Wijers, \& van Staveren, 2001; Eimer, 1993; Falkenstein, Hoormann, \& Hohnsbein, 1999; Gajewski \& Falkenstein, 2013; Pfefferbaum, Ford, Weller, \& Kopell, 1985; van Boxtel, van der Molen, Jennings, \& Brunia, 2001). Finally, the P3 related to positive feedback has been shown to be larger in highdemand tasks, and thus it has been suggested that reward feedback is more valued when subjects had to put in a lot of effort (Ma, Meng, Wang, \& Shen, 2014; Schevernels et al., 2014). Thus, we expected larger P3 amplitudes evoked by correct feedback in go trials than in no-go trials. Together, with this study we sought to use ERPs related to the cue, target, and feedback to investigate whether the action-dominated activity as found by Guitart-Masip et al. (2011) and Guitart-Masip, Chowdhury et al. (2012) might also partly arise from differences in preparatory mental effort.

\section{Materials and Method}

\section{Participants}

Twenty-one subjects participated in the experiment (17 women, $M$ age $=25.2$ years, $S D$ age $=5.4$ years $)$. They were all righthanded, had correct or corrected-to-normal vision, and reported no history of psychiatric or neurological disorders. Prior to participation, written informed consent was obtained according to the procedure approved by the local ethics committee. After finishing the task, each subject received a financial compensation of $25 €$ plus an additional bonus depending on their task performance (maximal 10€).

\section{Paradigm}

In the present study, we used a modified version of the orthogonalized go/no-go task of Guitart-Masip et al. (2011). In this experiment, cues indicate whether the upcoming trial will be a go or nogo trial. In go trials, subjects have to respond as quickly as possible according to the location of the target (left or right), while in no-go trials no button has to be pressed when the target appears. Cues also indicate the potential valence of the trial (i.e., the outcome depending on task performance). In addition to the two original valence-related cues (reward and punishment), we included neutral 


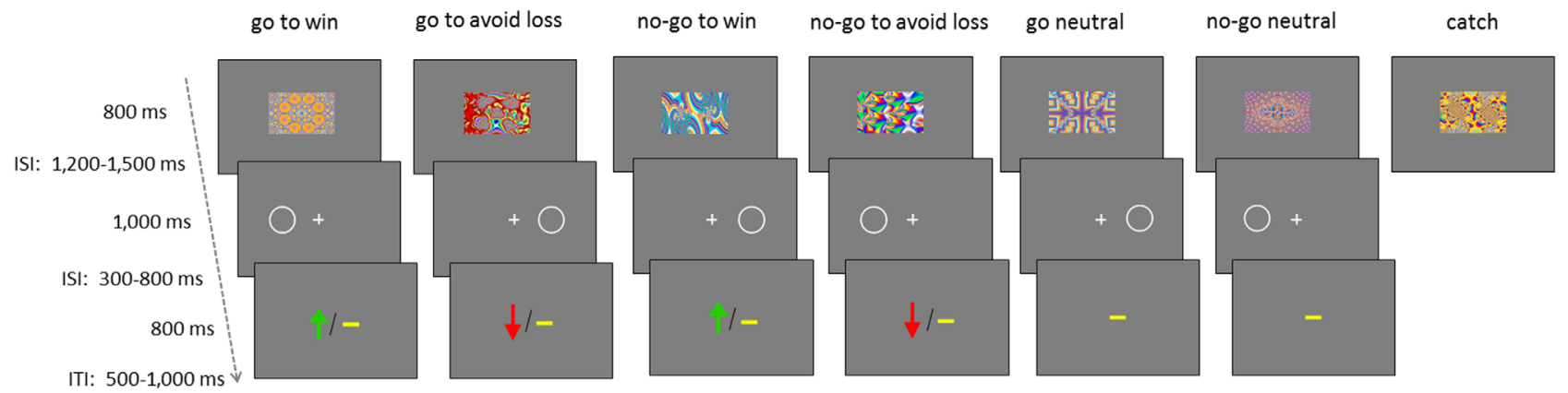

Figure 1. Task procedure. At the start of each trial, a cue signaled the possible value of the trial (win, avoid losing, or neutral) and indicated whether the subject would have to respond (go trials) or not (no-go trials) to the upcoming target. In go trials, subjects had to press a button according to the location of the target. In cued catch trials, subjects knew that no target would be presented after the cue. At the end of each noncatch trial, feedback was presented dependent on the trial type and performance. A green arrow pointing upwards meant that subjects won 10 eurocents, while a red arrow pointing downwards indicated a 10 eurocents loss. A yellow horizontal bar signaled that no money was won or lost. The feedback was probabilistic so that only $70 \%$ of correct and fast responses in go trials and 70\% of correct withholding responses in no-go trials were rewarded (win trials) or not punished (avoid-losing trials).

cues in which performance outcome was not related to reward or punishment to be able to further investigate motivational effects by comparing against a neutral baseline. Moreover, we implemented cued catch trials in which participants knew that no target would be presented, and which therefore should not trigger any preparatory activity. Except for catch trials, participants received feedback about the outcome at the end of each trial. Stimulus presentation durations and timing differed from the original experiment since they were optimized for an EEG setting (see Figure 1). In contrast to the studies of Guitart-Masip et al. (2011) and Guitart-Masip, Chowdhury et al. (2012), who used 50\% cue-only trials in order to disentangle hemodynamic activity related to cue processing from subsequent processes, the high temporal resolution of EEG (combined with the relatively slow succession of events) allows us to distinguish motor-related from preparatory-related activity directly, hence largely abolishing the need for this procedure.

Different fractal images $\left(5^{\circ} \times 7^{\circ}\right)$ served as cues indicating the trial type. We kept the four conditions implemented in the study of Guitart-Masip et al. (2011): respond quickly and correctly to obtain a reward (go to win), respond quickly and correctly to avoid punishment (go to avoid losing), do not respond to obtain a reward (no-go to win), and do not respond to avoid punishment (no-go to avoid losing). Furthermore, three other trial types were included serving as baseline conditions: respond quickly and correctly although there is no prospect of reward or punishment (go neutral), do not respond although there is no prospect of reward or punishment (no-go neutral), and attentively wait for the next cue since no target will appear (cued catch). Hence, in total there were seven different fractal images. Each fractal cue was presented for $800 \mathrm{~ms}$ and occurred for an equal number of times.

After a variable delay of 1,200 to $1,500 \mathrm{~ms}$ (only showing a fixation cross), the next cue appeared in cued catch trials and a target was presented for $1,000 \mathrm{~ms}$ in noncatch trials. Targets were circles (radius $3^{\circ}$ ) that were randomly displayed on the left or right side $\left(5^{\circ}\right)$ of a central fixation cross. In go trials, subjects had to press the letter A with their left index finger when the circle was displayed on the left side of the screen while they had to press the letter L with their right index finger when the circle was shown on the right side of the screen (QWERTY-layout keyboard). Moreover, in go trials responses had to be made quickly since a fixed response time-out was implemented (which was not the case for no-go trials). This time-out varied between subjects since it was based on their performance in the second training run (see below). More spe- cifically, the maximal time to respond in go trials was defined by taking the average of response times on correct go trials in this training run plus $50 \mathrm{~ms}{ }^{1}$

At the end of each noncatch trial, feedback was presented for $800 \mathrm{~ms}$ following a varying interstimulus interval of 300 to 800 ms. A green upward-pointing arrow indicated a gain of 10 eurocents, a red downward-pointing arrow signaled a 10 eurocents loss, and a yellow horizontal bar was shown when subjects did not lose or win any money. The outcome was dependent on the trial type as well as on the subject's performance. However, consistent with the paradigm of Guitart-Masip et al. (2011) and Guitart-Masip, Chowdhury et al. (2012), in win and avoid-losing conditions, the outcome was furthermore probabilistic in the sense that even when subjects performed the task correctly only $70 \%$ of correct reactions in win trials were rewarded and only $70 \%$ of correct reactions in avoid-losing trials were not punished. Hence, in $70 \%$ of the trials, subjects would gain a reward in win trials and avoid punishment in avoid-losing trials when they gave a fast and accurate response in go conditions and withheld their response in no-go conditions. In neutral task conditions, a horizontal bar was always presented indicating that no money had been won or lost.

Before starting the experiment, task requirements were explained and subjects were instructed to minimize eye movements and blinking. Subjects were explicitly told that they could win extra money contingent on their task performance, and they were informed about the probabilistic feedback. Furthermore subjects had to memorize the meaning of all seven cues, which was also repeated at the beginning of every experimental run. To ensure that subjects had learned the cue meanings, we implemented a first practice session including 10 trials per condition (70 trials in total) in which neither a response time-out nor a probabilistic outcome was applied, and the Dutch words goed (correct) or fout (incorrect) were presented to provide additional feedback. If accuracy was worse than $75 \%$ in one of the conditions, we restarted this practice block. Subsequently, subjects performed a second practice run in which trials were presented in the same way as in the experimental task (with probabilistic outcome). In the experimental session, seven runs of 70 trials were performed with 10 trials per condition

1. We implemented this subject-based time-out instead of a fixed maximum response time of $700 \mathrm{~ms}$ (Guitart-Masip et al., 2011), which turned out to be too long during piloting this task in order to assure that similar accuracy levels would be reached as in the study of GuitartMasip and colleagues. 
presented equally often in a randomized order. This resulted in a total of 490 trials with 70 trials per condition. In each run, a selfpaced break was implemented after 35 trials. Moreover, after every run subjects were informed about the amount of money they had already won. Two versions of the experiment were created to counterbalance ${ }^{2}$ the meaning of the fractal cues across participants.

\section{EEG Acquisition and Preprocessing}

EEG signals were recorded using a BioSemi ActiveTwo system (common mode sense-driven right leg [CMS-DRL] reference) and sampled at $256 \mathrm{~Hz}$. Data were recorded from $64 \mathrm{Ag}-\mathrm{AgCl}$ scalp electrodes positioned according to the International 10-20 location configuration. External electrodes were attached to the left and right mastoid for offline rereferencing. Horizontal and vertical eye movements were measured using electrodes placed at the external canthus of the left and right eye and directly above and below the left eye, respectively. Subjects were tested in a dimly lit, electrically shielded room.

A combination of EEGLAB (Delorme \& Makeig, 2004) and ERPLAB (Lopez-Calderon \& Luck, 2014) was used to analyze the collected EEG data. As a first step, we rereferenced the data to the average of the left and right mastoid. Subsequently, data was lowpass filtered $(30 \mathrm{~Hz})$, and eye blinks were removed using independent component analysis. Channels that were very noisy were interpolated (only six channels in total across all datasets). Baselinecorrected epochs were created time-locked to the onset of the cue, target, and feedback with a time window of $-200 \mathrm{~ms}$ to $2,300 \mathrm{~ms}$, $-200 \mathrm{~ms}$ to $1,000 \mathrm{~ms}$, and $-200 \mathrm{~ms}$ to $800 \mathrm{~ms}$, respectively. Epochs containing eye movements (step function with threshold 60 $\mu \mathrm{V}$ and window size $400 \mathrm{~ms}$ in the bipolar horizontal electrooculogram channel) and extreme values in scalp electrodes $(> \pm 150$ $\mu \mathrm{V}$ ) were automatically removed, and afterwards all epochs were visually inspected for possible additional missed artifacts. This resulted on average in $4 \%$ rejected cue-locked epochs, $2 \%$ rejected target-locked epochs, and $2 \%$ rejected feedback-locked epochs.

\section{Data Analysis}

Behavior. Accuracy, that is, a correct button press within the response time-out window in go trials and not responding in no-go trials, was investigated using a repeated measures analysis of variance (rANOVA) with within-subject factors action (go/no-go) and valence (win/avoid losing/neutral). In case Mauchly's test indicated that the assumption of sphericity did not hold, degrees of freedom were corrected using the Greenhouse-Geisser estimates of sphericity. The number of reactions that exceeded the response time-out and reaction times in correct go trials (i.e., only including reaction times within the response window) were analyzed using a rANOVA with the factor valence (win/avoid losing/neutral) and post hoc paired-sample $t$ tests. If tests were significant, we also report effect sizes (Cohen's $d$ and partial eta squared, $\eta_{\mathrm{p}}{ }^{2}$ ).

2. More specifically, the first version of the counterbalancing is described in Figure 1. In the second version, we crossed cue meanings for action and valence conditions so that fractal images that would indicate go to win and go to avoid losing in Version 1 would indicate no-go to avoid losing and no-go to win respectively in Version 2, while no-go to win and no-go to avoid-losing images in Version 1 would indicate go to avoid losing and go to win respectively in the second version. Furthermore, neutral images that indicated go in the first version indicated (neutral) no-go in the second version and the other way around.
ERPs. For both cue-locked and target-locked ERPs, mean amplitude measurements were derived within a specific time range and region of interest. ERPs related to cues and targets only included trials in which subjects responded accurately (i.e., within time-out window for go trials). Feedback-related analyses included trials in which responses and feedback were correct. ${ }^{3}$ All analyses were performed on $30 \mathrm{~Hz}$ low-pass filtered data, but for illustration purposes a $15 \mathrm{~Hz}$ low-pass filter was applied in the figures. Amplitudes were analyzed using rANOVAs with the factors action (go/ no-go) and valence (win/avoid losing/neutral) and applying the Greenhouse-Geisser degrees of freedom correction whenever necessary. Hence, if Mauchly's test of sphericity was significant, we report uncorrected $F$ values, Greenhouse-Geisser corrected $p$ values, and epsilon $(\varepsilon)$. Post hoc tests were performed to further investigate significant effects. Effect sizes (Cohen's $d$ and partial eta squared) are reported when statistical results were significant. ERPs related to cued catch trials were not included in the statistical analyses, but they are displayed in the figures as a reference.

The parietal cue-evoked P3 was maximal at electrode sites Pz, $\mathrm{P} 1$, and P2 between 400 and $600 \mathrm{~ms}$ after cue onset (mainly for go trials). Subsequently a large negative component, (i.e., the CNV) was detected between 1,000 and 2,000 ms (earliest onset of the target) mainly in go conditions at frontocentral locations (FCz, FC1, $\mathrm{FC} 2, \mathrm{Cz}, \mathrm{C} 1$, and C2). Consistent with earlier studies (Broyd et al., 2012; Goldstein et al., 2006; Jonkman, Lansbergen, \& Stauder, 2003; Schevernels et al., 2014), this prolonged negativity was separated in an early and late $\mathrm{CNV}$ from 1,000 to $1,500 \mathrm{~ms}$ and 1,500 to $2,000 \mathrm{~ms}$, respectively.

Since we were also interested in early effects of different kinds of cues on visual processing of the target circle, we also analyzed different target-related components. Early positive (P1) and negative waves (N1) were maximal in posterior regions on the contralateral side of target presentation. Hence, P1 and N1 components were observed in PO7 when the circle was presented on the right and in PO8 when the circle was presented on the left side of the screen, within a time window of 80 to $120 \mathrm{~ms}$ for P1 and 140 to $180 \mathrm{~ms}$ for N1. Also, a clear broad centroparietal P3 was quantified between 250 and $400 \mathrm{~ms}$ at centroparietal (CPz, CP1, and CP2) and parietal electrode sites (Pz, P1, and P2). We also explored the inhibition-related frontal N2/P3 complex, yet we could not clearly detect these components in no-go trials, and accordingly they were not analyzed statistically. The processing of correct feedback gave rise to a prominent $\mathrm{P} 3$ that was maximal between 300 and $450 \mathrm{~ms}$ at parietal electrode sites (Pz, P1, P2, POz, PO3, PO4).

\section{Results}

\section{Behavior}

Analyses related to behavioral accuracy revealed a significant main effect of action, $F(1,20)=42.48, p<.001, \eta_{\mathrm{p}}{ }^{2}=.68$, showing higher accuracy for no-go trials compared to go trials. ${ }^{4}$ However, although reaching similar overall accuracy rates, we failed to replicate an important feature of the behavioral results of Guitart-Masip

3. Feedback related to erroneous or too-late responses was excluded due to very low error rates especially in no-go trials, and analyses did not encompass incorrect feedback to correct responses (due to the probabilistic aspect) to minimize effects of expectancy violation.

4. In go trials, subjects only very sporadically responded with the wrong button before the time-out (on average in less than $0.5 \%$ of the trials). Hence, in the current paradigm, "errors" in go trials mostly encompassed too-slow responses (average of 12\%). 
Table 1. Behavioral Performance

\begin{tabular}{lccc}
\hline \hline & Win & Avoid loss & Neutral \\
\hline Accuracy, $\%$ & & & \\
$\quad$ Go trials & $89(2.1)$ & $88(1.8)$ & $86(2.4)$ \\
$\quad$ No-go trials & $100(0.1)$ & $99(0.2)$ & $100(0.2)$ \\
$\begin{array}{l}\text { Reaction time, ms } \\
\text { Go trials }\end{array}$ & $316.6(7.3)$ & $318.6(6.4)$ & $325.4(7)$ \\
\hline \hline
\end{tabular}

Note. Mean values and standard errors of the mean (within brackets) are shown for all conditions.

et al. (2011) in that we neither found a significant main effect of valence nor a significant interaction (both $p s>.15$ ). This pattern also didn't change when including responses that exceeded the response time-out window seeing that the number of go trials that exceeded the maximal response time was similar for different types of valence conditions $(p>.15)$. In contrast, in correct in-time go trials, we did find an effect of valence, $F(2,40)=8.22, p=.001$, $\eta_{\mathrm{p}}{ }^{2}=.29$. Specifically, subjects responded slower in the neutral condition compared to the avoid-losing, $t(20)=2.95, p=.008$, $d=.22$, and win condition, $t(20)=4.63, p<.001, d=.27$, but reaction times in the latter two conditions did not differ $(p>.4)$. These behavioral results are summarized in Table 1 .

\section{ERPs}

Cue-locked ERPs. The cue-locked P3 amplitude was considerably larger for cues signaling go trials, $F(1,20)=57.36, p<.001$, $\eta_{\mathrm{p}}{ }^{2}=.74 ; M=10.05, S D=5.09$ versus $M=4.18, S D=4.36$ (see Figure 2). Also, a main effect of valence was observed, $F(2,40)=4.26, p=.021, \eta_{\mathrm{p}}{ }^{2}=.18$, but valence also significantly interacted with action, $F(2,40)=3.67, p=.048, \varepsilon=.77, \eta_{\mathrm{p}}{ }^{2}=.16$, due to a larger P3 amplitude in go to win trials compared to go to avoid-losing trials, $t(20)=4.07, p=.001, d=.36 ; M=11.64$,

A

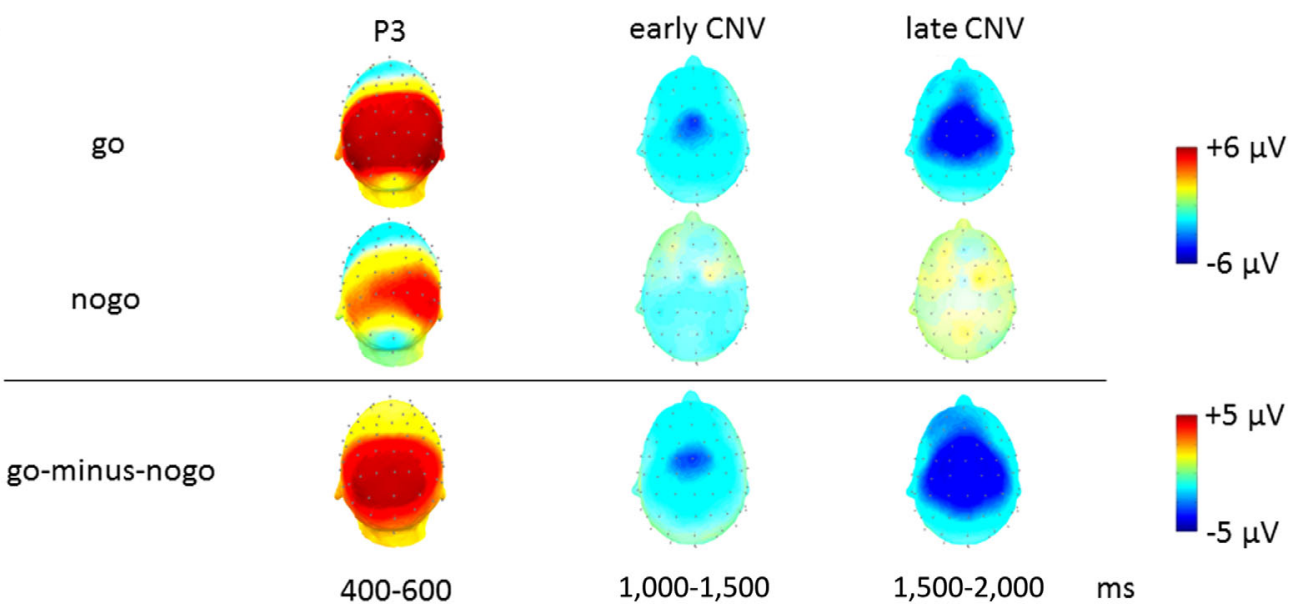

B

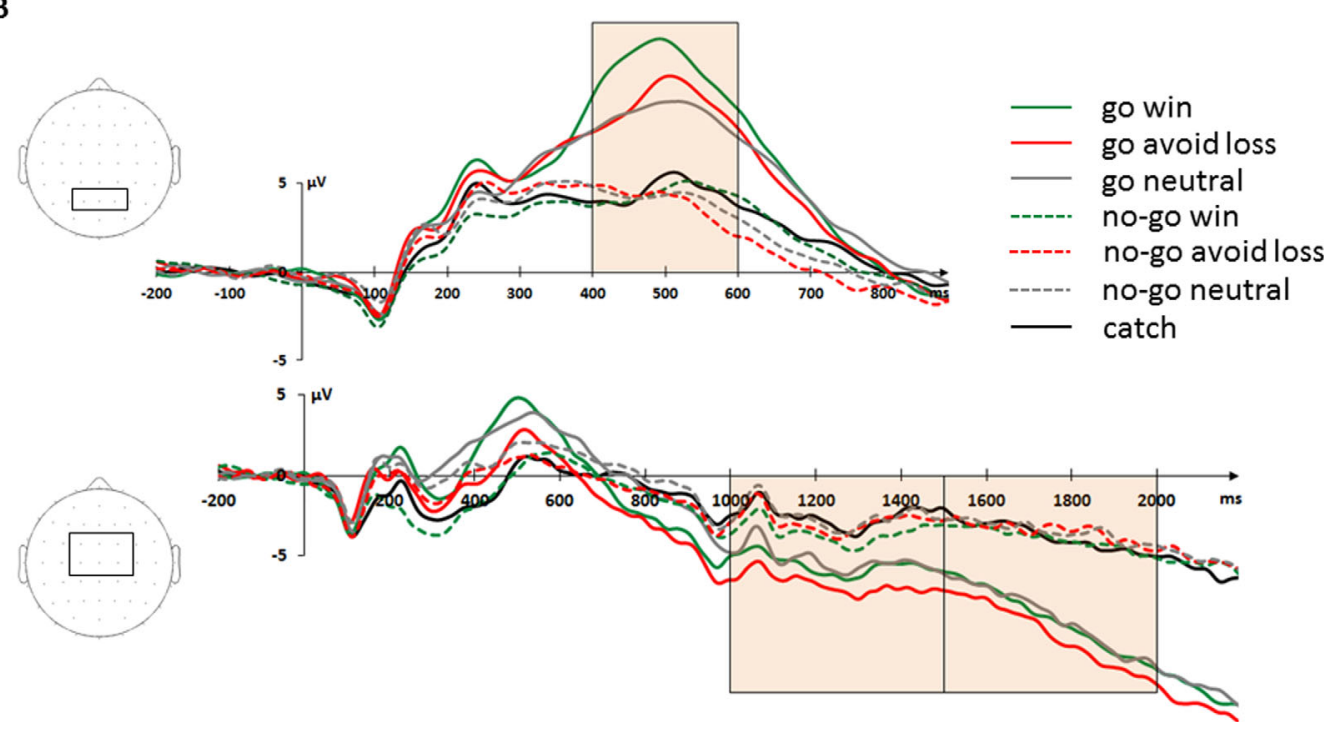

Figure 2. Cue-locked ERP results. A: Topographical maps reflecting the P3, early CNV, and late CNV in go and no-go trials, and the corresponding differences indicating the main effect of action. Note that the topographical maps of the (early and late) CNV in go and no-go trials reflect the difference between go or no-go trials on one hand and cued catch trials (which should not induce any preparatory activity) on the other hand. Go trials elicited larger positivities than nogo trials between 400 and $600 \mathrm{~ms}$ (P3). Moreover, in go trials a clear (early and late) CNV is observed, which is not the case for no-go trials. B: Grandaveraged ERP waves locked to the cue over electrode sites Pz, P1, and P2 show larger P3 amplitudes in go trials and especially in go to win trials compared to go to avoid-losing and neutral trials. Moreover, grand-averaged waveforms over frontocentral sites (FCz, FC1, FC2, Cz, C1, and C2) are depicted for each condition, reflecting the early and late CNV component in go trials. Frames indicate the time range of the different components (as included in the analyses). 
$S D=5.74$ versus $M=9.56, S D=5.7$, and go neutral trials, $t(20)=3.31, \quad p=.004, \quad d=.5 ; \quad M=11.64, \quad S D=5.74 \quad$ versus $M=8.95, S D=4.91$, but a similar P3 amplitude in the different no-go conditions ( $p$ s $>.4$; see Figure $2 \mathrm{~B}$ ). Both early and late CNV amplitude showed a clear main effect of action, $F(1,20)=11.8$, $p=.003, \eta_{\mathrm{p}}{ }^{2}=.37$ and $F(1,20)=21.81, p<.001, \eta_{\mathrm{p}}{ }^{2}=.52$, with larger negative-going waves for cues signaling go trials $(M=-5.85, S D=3$ vs. $M=-2.96, S D=2.74$ and $M=-20.64$, $S D=5.09$ vs. $M=-5.89, S D=3.38$, respectively; see Figure 2), whereas neither the main effect of valence nor the interaction between the factors was significant (all $p \mathrm{~s}>.1$ ).

Target-locked ERPs. The posterior target-locked P1 did not show any significant differences $(p s>.2)$, but the visual N1 was larger in go trials compared to no-go trials, $F(1,20)=11.48, p=.003$, $\eta_{\mathrm{p}}{ }^{2}=.37 ; M=-1.42, S D=3.38$ versus $M=-0.32, S D=2.69$ (see Figure 3A). We did not find a significant main effect of valence $(p>.4)$ nor a significant interaction $(p>.1)$ related to the N1 component. The target-locked parietal P3 amplitude showed a large main effect of action, $F(1,20)=134.73, p<.001, \eta_{\mathrm{p}}{ }^{2}=.87$, and an interaction between action and valence, $F(2,40)=3.15, p=.054$, $\eta_{\mathrm{p}}{ }^{2}=.14$. Figure $3 \mathrm{~A}$ illustrates that these effects reflect significantly larger P3 amplitudes in go trials and especially in win and avoidlosing trials compared to neutral trials, $t(20)=3.54, p=.002$, $d=.29 ; M=12.02, S D=5.04$ versus $M=10.6, S D=4.62$; and $t(20)=2.1, \quad p=.05, \quad d=.22 ; \quad M=11.62, \quad S D=4.73 \quad$ versus $M=10.6, S D=4.62$, respectively, while there was no difference between the no-go conditions (all $p s>.15$ ). Results shown in Figure $3 \mathrm{~B}$ indicate that the typical N2/P3 complex was not observed in nogo trials. These inhibition-related N2 and P3 components are usually detected at frontal sites between approximately 200 and $300 \mathrm{~ms}$ and 300 and 500 ms, respectively (see, e.g., Bokura et al., 2001; Falkenstein et al., 1999; Smith et al., 2007) with larger amplitudes in no-go trials compared to go trials. Concerning the N2, there was no clear negative component between 200 and $300 \mathrm{~ms}$ aftertarget presentation, and although ERPs between 200 and $300 \mathrm{~ms}$ elicited by targets in no-go trials showed a less positive wave than target-evoked ERPs in go trials, the distribution was more parietal than frontal (see Figure $3 \mathrm{~B}$ ). Also, importantly, the subsequent time range showed no indication of an enhanced frontal positivity in no-go trials (between 300 and $500 \mathrm{~ms}$ ). Rather, we only detected a broad centroparietal positivity starting around $200 \mathrm{~ms}$ and lasting until $500 \mathrm{~ms}$ that was larger in go trials likely reflecting the parietal P3 described earlier (depicted by a larger negativity when subtracting waves related to go trials from no-go trials).

Feedback-locked ERPs. The correct feedback-evoked P3 showed a significant main effect of the factors action, $F(1,20)=9.55$, $p=.006, \quad \eta_{\mathrm{p}}{ }^{2}=.32$, and valence, $F(2,40)=39.19, \quad p<.001$, $\eta_{\mathrm{p}}{ }^{2}=.67$, but also a significant interaction, $F(2,40)=3.92$, $p=.028, \eta_{\mathrm{p}}{ }^{2}=.16$. As can be observed in Figure 4, win feedback (arrow pointing upwards) elicited the largest P3s when subjects had to perform an action. Moreover, the P3 amplitude related to positive feedback was significantly larger in go compared to no-go trials not

A
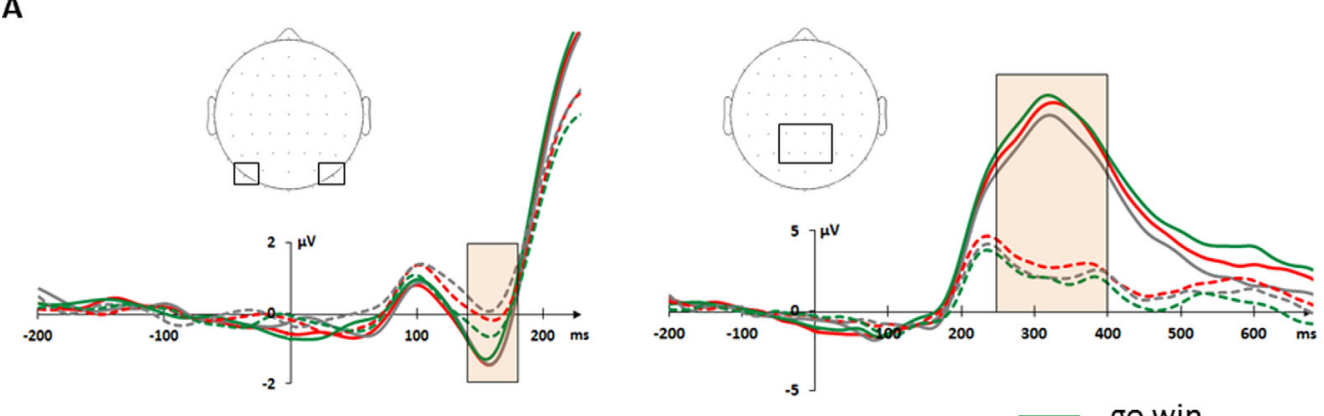

B

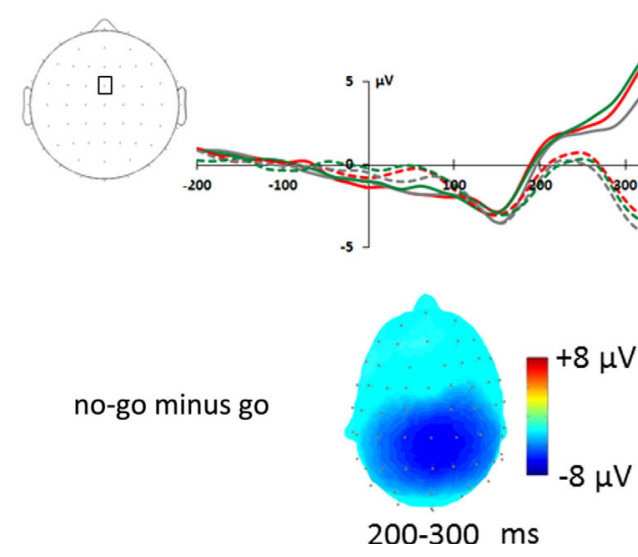

go win go avoid loss go neutral no-go win no-go avoid loss no-go neutral

Figure 3. A: Target-locked ERPs. Waveforms averaged over PO7 for targets presented on the right side of the screen and PO8 for targets presented on the left side of the screen, showing enhanced target-locked N1 in go trials (left). Grand-averaged target-locked ERPs over (centro)parietal regions (Pz, CPz, P1, CP1, P2, and CP2) reflecting the target P3 between 250 and $400 \mathrm{~ms}$, illustrating enhanced P3 amplitudes in go trials and especially in go to win and go to avoid-losing trials (right). B: Absence of the no-go N2/P3 complex. Waveforms are shown at frontal electrode site FCz where typically a larger negativity between 200 and $300 \mathrm{~ms}$ (N2) and enhanced positivity between 300 and $500 \mathrm{~ms}$ (P3) in no-go trials compared to go trials is observed. As can also be observed in the topographical maps depicting the difference between no-go and go trials at different time points, no-go trials did not display an enhanced negativity at frontal electrode sites, but rather go trials showed a broad (centroparietal) enhanced positivity (here reflected by a larger negativity in the no-go minus go difference). 


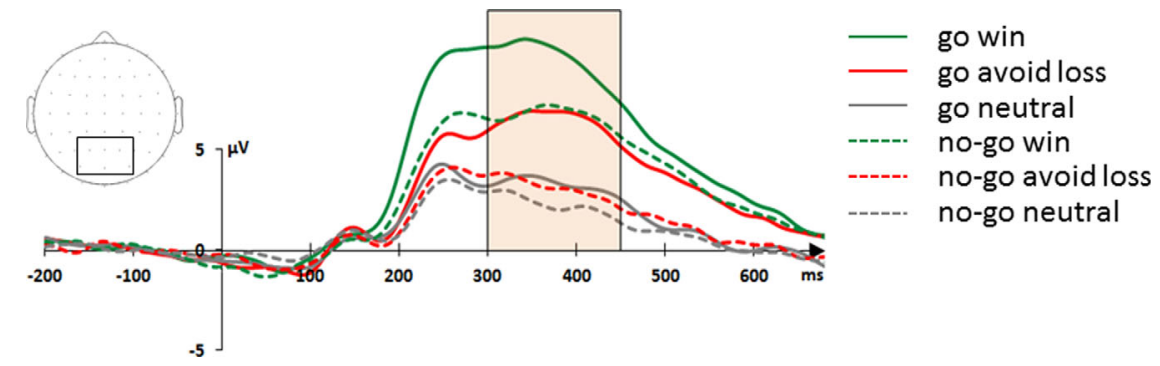

Figure 4. Correct feedback-locked ERPs. Over parietal regions (Pz, P1, P2, POz, PO3, and PO4), grand-averaged ERPs show more positive P3 components evoked by correct feedback in go trials compared to no-go trials only in the go to win and avoid-losing conditions. Frames indicate the time range of the different components (as included in the analyses).

only in the win condition (arrow pointing upwards), $t(20)=2.93$, $p=.008, d=.55 ; M=9.58, S D=6.21$ vs. $M=6.74, S D=3.81$, but also in the avoid-losing condition (horizontal bar), $t(20)=2.93$, $p=.008, d=.61 ; M=6.57, S D=6.96$ vs. $M=3.14, S D=3.82$. On the other hand, the P3 was smallest for correct feedback in neutral trials, and it did not significantly differ between neutral go and no-go trials $(p>.1)$. To further identify effects of valence, we also compared trials with a different outcome value to correct responses in go and no-go trials, respectively. We found that, when subjects had to respond to the target (go trials), the correct-feedback P3 amplitude was increased from win trials to avoid-losing trials, $t(20)=3.93, \quad p=.001, \quad d=.46 ; \quad M=9.58, \quad S D=6.21 \quad$ versus $M=6.57, S D=6.96$; to neutral trials, $t(20)=2.93, p=.008$, $d=.58 ; M=6.57, S D=6.96$ versus $M=3.31, S D=3.75$; while a similar pattern was found for no-go trials with a larger feedback positivity in win trials compared to avoid-losing trials, $t(20)=5.47$, $p=.001, \quad d=.94 ; \quad M=6.74, \quad S D=3.81$ versus $M=3.14$, $S D=3.82$, and a larger amplitude in no-go to avoid-losing trials than in no-go neutral trials, but this latter effect was smaller than in go trials and was only borderline significant, $t(20)=1.89, p=.074$, $d=.23 ; M=3.14, S D=3.82$ versus $M=2.31, S D=3.34$.

\section{Discussion}

The goal of the current study was to investigate differential involvement of (preparatory) mental effort in the orthogonalized go/no-go task of Guitart-Masip et al. (2011) and Guitart-Masip, Chowdhury et al. (2012) using ERPs. In line with the idea that preparing for a go trial is more demanding than preparing for a no-go trial, we found diminished cue-evoked P3 amplitudes and an absence of the preparation-related CNV component for the expectation of no-go targets. Moreover, visual attention to the target was decreased in no-go trials as indicated by smaller N1 and P3 amplitudes. Finally, the P3 amplitude related to correct feedback was significantly larger in motivated (win and avoid-losing) go trials, which might suggest more positive subjective appraisal of correct feedback for a more effortful task. All these results together point in the direction of differential cognitive resource engagement during preparation, task performance, and feedback evaluation in anticipated go trials compared to anticipated no-go trials.

\section{Main Effects of Action and Effort Anticipation}

The key focus of our experiment lies in the manipulation of action, and in line with the effect found by Guitart-Masip et al. (2011) and Guitart-Masip, Chowdhury et al. (2012), we observed higher accuracy in no-go trials (nearly $100 \%$ correct) suggesting that these trials were less difficult than go trials. Moreover, the cue-locked P3 as well as the CNV component showed highly significant effects of action with larger amplitudes for the anticipation of go trials compared to no-go trials. The P3 has been suggested to reflect activity in the locus coeruleus-norepinephrine (LC-NE) system that is involved in stimulus evaluation and decision making, thereby guiding attention allocation in favor of goal-relevant stimuli (Nieuwenhuis, Aston-Jones, \& Cohen, 2005). Hence, the large difference in P3 amplitude between go and no-go trials might suggest that go trials are identified as being more task relevant, promoting more attention to the go stimuli.

More importantly, we did find a substantially larger early and late $\mathrm{CNV}$ in go trials than in no-go trials. For the latter, we in fact found that the CNV was practically absent (compared to catch trials), in line with previous findings showing a significant cuerelated action effect (Filipović et al., 2001; Funderud et al., 2012; Randall \& Smith, 2011; Rosahl \& Knight, 1995). This difference probably entails more motoric preparation but also more cognitive preparation in go trials considering that it has been shown that the CNV also or mainly reflects effortful cognitive preparation processes, and that participants in the current task cannot prepare for a specific response yet (Ansari \& Derakshan, 2011; Cui et al., 2000; Falkenstein, Hoormann, Hohnsbein, \& Kleinsorge, 2003; Gómez, Flores, \& Ledesma, 2007; Lorist et al., 2000; Rösler et al., 1997; Wild-Wall, Hohnsbein, \& Falkenstein, 2007). Hence, these results are in favor of our hypothesis that no-go cues differ from go cues in mental preparatory activation going beyond merely motor preparation. Importantly, this effect could very well link back to the action-dominated activity in the dopaminergic source and target regions found by Guitart-Masip et al. (2011) and Guitart-Masip, Chowdhury et al. (2012) given evidence for a link between the CNV and central dopaminergic activity ${ }^{5}$ (Amabile et al., 1986; Fan et al., 2007; Gerschlager et al., 1999; Linssen et al., 2011; Oishi, Mochizuki, Du, \& Takasu, 1995), and the fact that these areas have been found to be sensitive to differences in preparatory effort (e.g., Boehler et al., 2011; see also below). We note that the modest amount of jittering used here between the cue and the target might have some implications for the interpretation of the CNV (in terms of temporal anticipation). Yet, having some jitter increases the similarity to the original paradigm of Guitart-Masip et al. that used an extensive amount of jittering as is typical in fMRI studies. Yet, we shortened the jittering period extensively (to an interval of 1,200 to $1,500 \mathrm{~ms}$ after cue offset), which means subjects expect the target on average in a brief time window starting 1,200 ms after cue offset. Importantly, we limited our analysis to the time window

5. Of course, the CNV does not directly signal subcortical dopamine release (Cohen, Cavanagh, \& Slagter, 2011), but it could rather reflect its effects on cortical brain areas via dopaminergic pathways. 
preceding the earliest time point of target presentation. Moreover, the similarly large action effects across both early and late portions of the CNV suggest a sustained anticipation effect of around $1 \mathrm{~s}$ in duration in anticipated go trials.

The target-related ERPs provided further evidence for differences in effort-related processes. Specifically, we found a diminished contralateral posterior N1 component in no-go trials, implying decreased early visual attention to the circle target when subjects did not have to perform an action (and thus did not need to discriminate the target's location; see, e.g., Vogel \& Luck, 2000). In addition, the subsequent parietal P3 amplitude was enhanced in go trials compared to no-go trials consistent with ERP results found in studies using the go/no-go task showing a more parietal scalp distribution for go trials (Bokura et al., 2001; Bruin \& Wijers, 2002; Liu, Xiao, Shi, \& Zhao, 2011; Tekok-Kilic, Shucard, \& Shucard, 2001). The parietal go P3 component has been distinguished from the frontal no-go P3 (Bokura et al., 2001; Pfefferbaum \& Ford, 1988; Tekok-Kilic et al., 2001) with the first being more similar to the classic P300, likely reflecting target detection and evaluation, response production, and executive control processes (Eimer, 1993; Liu et al., 2011; Pfefferbaum et al., 1985; Tekok-Kilic et al., 2001). Remarkably, in no-go trials we did not observe any typical inhibition-related components during target processing like the no-go frontal N2/P3 complex (see Figure 3B; Bokura et al., 2001; Bruin et al., 2001; Eimer, 1993; Falkenstein et al., 1999; Gajewski \& Falkenstein, 2013; Kok, 1986; Pfefferbaum et al., 1985; Randall \& Smith, 2011; Smith, Johnstone, \& Barry, 2008), suggesting that there is little active inhibition involved in cued no-go trials.

Moreover, correct feedback elicited a parietal P3 that was more positive in go trials than in no-go trials in both win and avoidlosing conditions but not in neutral conditions, which implies an effect of action when motivation is high. Considering that the feedback P3 is involved in high-level affective evaluation of the outcome (Nieuwenhuis et al., 2005; Wu \& Zhou, 2009; Zhou, Yu, \& Zhou, 2010), studies that found enhanced correct-feedback P3 amplitudes in more difficult tasks have explained this in terms of more positive evaluation of the feedback stimulus after one had to put in more effort to correctly perform the task (Ma et al., 2014; Schevernels et al., 2014), which is again consistent with the notion that effort differed between cued go and no-go trials in the present experiment. However, the amplitude of the feedback P3 (and the feedback negativity) has been found to be enhanced when feedback is contingent upon a performed action (Yeung, Holroyd, \& Cohen, 2005). In the current task, action in go trials is equally contingent upon the outcome as is inaction in no-go trials, and thus the feedback P3 might be similarly enhanced in go and no-go conditions according to this idea, but it is conceivable that actual acting plays a role in such processes, too. Furthermore, the feedback-related P3 amplitude has also been shown to be enhanced when events are unlikely or unexpected (Bellebaum \& Daum, 2008; Hajcak, Holroyd, Moser, \& Simons, 2005; Hajcak, Moser, Holroyd, \& Simons, 2007; Wu \& Zhou, 2009). In the current task, accuracy was lower in go trials compared to no-go trials and thus correct feedback could be experienced as more unexpected. However, the correct-feedback P3 did not significantly differ between go trials and no-go trials in neutral conditions for which the frequency proportion is similar as in win and avoid-losing conditions. Furthermore, the subjective frequency difference might have been reduced given that $30 \%$ of correct responses were not rewarded or punished in both go and no-go trials (probabilistic feedback). Hence, we favor the stimulus-evaluation over the expectancy account to explain the action-related differences in correct-feedback P3 amplitudes in the current task. However, the value of an outcome is defined as a function of expectations. Given the probabilistic nature of the feedback, as also implemented by Guitart-Masip (2011) and Guitart-Masip, Chowdhury et al. (2012), these expectations are not easily characterized, and it is not clear how strongly people rely on the given feedback. Hence, the current paradigm is not optimally set up to identify brain responses to outcomes, and the correct P3 amplitude might very well be affected by factors other than just effort-related differences such as expectancy and action-outcome contingency.

Thus, the enhanced activity in the SN/VTA and the striatum in expected go trials compared to no-go trials that was found by Guitart-Masip et al. (2011) and Guitart-Masip, Chowdhury et al. (2012) might not only represent anticipated action effects but also differences in anticipated mental effort or resource allocations. This is in line with previous findings demonstrating dopaminergic and striatal involvement in the (preparatory) control of cognitive resources in order to achieve goals and optimize behavioral outcomes (Boehler et al., 2011; Krebs et al., 2012; Nieoullon, 2002; Plichta et al., 2013; Vassena et al., 2014). Related to this idea, Kurniawan et al. (2013) implemented a very similar paradigm but they orthogonalized physical effort and valence in a pure go-trial context by showing fractal cues that indicated the required force with which subjects had to squeeze a handgrip (high effort or low effort) and the probability to win or lose money. They found increased BOLD signals when anticipating higher effort in the ACC and the dorsal striatum, two typical reward-related brain areas. Although in the current study we investigate mental effort and not physical effort (as in the study of Kurniawan et al., 2013), it has been shown that they partly rely on similar underlying mechanisms and brain structures (like the ventral striatum) that motivationally drive both task-specific systems (Schmidt, Lebreton, Cléry-Melin, Daunizeau, \& Pessiglione, 2012). Also, in many studies it has been advocated that dopaminergic activity is involved in motivated behavior and specifically in the exertion of effort to overcome work-related requirements or costs (Kurniawan, GuitartMasip, \& Dolan, 2011; Salamone \& Correa, 2012; Salamone, Correa, Farrar, \& Mingote, 2007; Salamone et al., 2005). However, whether this link between dopamine and effort can only be established when an action is required or whether it also plays a role in action inhibition or withdrawal is not clear. Hence, it would be interesting to manipulate (preparatory) task demands. Yet, in the current basic task design, there seems to be no feasible way to create more difficult anticipated no-go trials without lowering stopping probability below $100 \%$.

The contribution of effort-related processes aside, a direct coupling between action and valence could of course still exist (in which dopamine might be involved; see also Frank, Moustafa, Haughey, Curran, \& Hutchison, 2007; Frank et al., 2004). This has also been suggested by other previous studies investigating instrumental learning, where it was shown that subjects were more successful in learning to withhold a response (no-go) when anticipating punishment and to actively respond (go) when anticipating reward (Cavanagh et al., 2013; Chowdhury, Guitart-Masip, Lambert, Dolan, \& Düzel, 2013; Guitart-Masip, Huy et al., 2012; Guitart-Masip et al., 2014; Richter et al., 2014). Nevertheless, in the current study action and effort are clearly intertwined, and anticipation of no-go does not necessarily induce (preparation for) active inhibition or withdrawal, but rather altogether diminished (preparatory) cognitive resources devoted to the task. 


\section{Effects of Valence and Interaction Effects}

Behaviorally, we did not replicate the asymmetrical valence effect in that there was no significant difference in accuracy levels or reaction times between go to win and go to avoid-losing conditions (although numerically going in the expected direction). This might be due to an overall difference in reaction time, since in the current experiment subjects responded on average approximately $200 \mathrm{~ms}$ faster compared to the studies of Guitart-Masip et al. (2011) and Guitart-Masip, Chowdhury et al. (2012). The generally slower response speed in the previous studies might be explained by the fMRI versus EEG scanning environment, differences in task instructions, task design, or training levels of subjects. Moreover, differences in response time-out might be relevant. In particular, our time-out procedure might have been too strict and could have pushed subjects to respond very fast and therefore leaves a smaller margin for improvements over conditions. Moreover, the implementation of a neutral condition might have increased the motivational value of the avoid-losing condition to a similar level as the win condition, which is suggested by smaller reaction times on both go to win and go to avoid-losing trials compared to go neutral trials. Of note, similar behavioral results for potential monetary gain and potential monetary loss have been found previously (Carter, MacInnes, Huettel, \& Adcock, 2009; Engelmann, Damaraju, Padmala, \& Pessoa, 2009; but see Ivanov et al., 2012; Paschke et al., 2015; Potts, 2011). Given these differences, our failure to replicate the asymmetrical valence effect on go accuracy might very well not translate into a challenge of its validity (also note that on the neural level we did observe some interactions between action and valence, see below). Yet, we note that, although it would have been valuable to replicate the behavioral interaction effect found by Guitart-Masip et al. (2011) and GuitartMasip, Chowdhury et al. (2012), also to evaluate it with reference to our neutral conditions, this was not the main interest of the current research since we focused on an alternative effort-related hypothesis for explaining action dominance (i.e., the main effect of action) in anticipatory brain activity.

The cue-locked P3 amplitude in go trials was increased for win trials compared to avoid-losing and neutral trials, thereby confirming the sensitivity of this component to reward (Goldstein et al., 2006, 2008; Hughes et al., 2012; Pfabigan et al., 2014; Schevernels et al., 2014), particularly in cues signaling go trials (Kohls et al., 2011). However, although we expected to find a motivational effect on the preparatory CNV component (mainly in go trials), we did not find a significant amplitude difference between anticipated gain, loss, and neutral trials. Results of previous studies investigating the role of reward and/or punishment during target preparation are also inconsistent, with some studies reporting a lack of motiva- tional influences on the CNV amplitude (Broyd et al., 2012; Goldstein et al., 2006; Sobotka et al., 1992) but others finding larger CNV amplitudes for cues indicating reward and/or punishment availability (Hughes et al., 2012; Pfabigan et al., 2014; Schevernels et al., 2014; Vuillier et al., 2015). In contrast to the current paradigm, these previous studies, however, usually investigated reward effects on the CNV amplitude in classic monetary incentive delay tasks that always involve the execution of an action.

Regarding target processing, the current study shows that the target-evoked go P3 is also affected by motivation, with larger amplitudes in both punishment and reward conditions compared to neutral conditions. The target P3 has been previously shown to be sensitive to reward likely reflecting increased attention to the target when it is highly significant or motivationally relevant (Baines, Ruz, Rao, Denison, \& Nobre, 2011; Hughes et al., 2012; Krebs, Boehler, Appelbaum, \& Woldorff, 2013; Schevernels et al., 2014). Turning to feedback processing, the parietal P3 evoked by correct feedback showed a main effect of valence with a decrease in P3 amplitudes from win trials to avoid-losing trials to neutral trials (although the latter effect was slightly decreased in the no-go condition). This is consistent with previous studies that have observed larger P3 amplitudes for reward compared to no-reward feedback (Bellebaum, Polezzi, \& Daum, 2010; Hajcak et al., 2007; Sato et al., 2005; Schevernels et al., 2014) illustrating this component's sensitivity to the magnitude of the reward outcome and thus suggesting its involvement in motivated significance (for an overview, see San Martín, 2012).

\section{Conclusions}

Together, the present behavioral and ERP results pattern points in the direction of effort-related differences between go and no-go trials in the orthogonalized go/no-go paradigm of Guitart-Masip (2011) and Guitart-Masip, Chowdhury et al. (2012). Most importantly, the absence of a CNV in no-go trials suggests a lack of involvement of preparatory cognitive processes. In turn, early and late visual attention during target processing was decreased and no typical inhibition-related components were detected after no-go cues. Finally, even the feedback-related ERP results likely implied a role of effort in that correct feedback seemed to be valued more positively in go trials. Therefore, we conclude that the established dominance of anticipated action (over valence) in typical rewardrelated brain networks simultaneously entails differences in mental effort engagement. From a more general perspective, our results suggest that action- and effort-related factors are difficult to disentangle and raise the question whether action effects would still hold when the amount of (anticipated) effort is held constant.

\section{References}

Adcock, R. A., Thangavel, A., Whitfield-Gabrieli, S., Knutson, B., \& Gabrieli, J. D. E. (2006). Reward-motivated learning: Mesolimbic activation precedes memory formation. Neuron, 50, 507-517. doi: 10.1016/j.neuron.2006.03.036

Amabile, G., Fattapposta, F., Pozzessere, G., Albani, G., Sanarelli, L., Rizzo, P. A., \& Morocutti, C. (1986). Parkinson disease: Electrophysiological (CNV) analysis related to pharmacological treatment. Electroencephalography and Clinical Neurophysiology, 64, 521524.

Ansari, T. L., \& Derakshan, N. (2011). The neural correlates of cognitive effort in anxiety: Effects on processing efficiency. Biological Psychology, 86, 337-348. doi: 10.1016/j.biopsycho.2010. 12.013
Baines, S., Ruz, M., Rao, A., Denison, R., \& Nobre, A. C. (2011). Modulation of neural activity by motivational and spatial biases. Neuropsychologia 49, 2489-2497. doi: 10.1016/j.neuropsychologia.2011.04.029

Beck, S. M., Locke, H. S., Savine, A. C., Jimura, K., \& Braver, T. S. (2010). Primary and secondary rewards differentially modulate neural activity dynamics during working memory. PloS ONE, 5, e9251. doi: 10.1371/journal.pone.0009251

Bellebaum, C., \& Daum, I. (2008). Learning-related changes in reward expectancy are reflected in the feedback-related negativity. European Journal of Neuroscience, 27, 1823-1835. doi: 10.1111/j.1460-9568. 2008.06138.x

Bellebaum, C., Polezzi, D., \& Daum, I. (2010). It is less than you expected: The feedback-related negativity reflects violations of reward magnitude 
expectations. Neuropsychologia, 48, 3343-3350. doi: 10.1016/ j.neuropsychologia.2010.07.023

Birbaumer, N., Elbert, T., Canavan, A. G. M., \& Rockstroh, B. (1990). Slow potentials of the cerebral cortex and behavior. Physiological Reviews, 70, 1-41.

Boehler, C. N., Hopf, J.-M., Krebs, R. M., Stoppel, C. M., Schoenfeld, M. A., Heinze, H.-J., \& Noesselt, T. (2011). Task-load-dependent activation of dopaminergic midbrain areas in the absence of reward. Journal of Neuroscience, 31, 4955-4961. doi: 10.1523/JNEUROSCI.484510.2011

Bokura, H., Yamaguchi, S., \& Kobayashi, S. (2001). Electrophysiological correlates for response inhibition in a go/nogo task. Clinical Neurophysiology, 112, 2224-2232. doi: 10.1016/S1388-2457(01)00691-5

Broyd, S. J., Richards, H. J., Helps, S. K., Chronaki, G., Bamford, S., \& Sonuga-Barke, E. J. S. (2012). An electrophysiological monetary incentive delay (e-MID) task: A way to decompose the different components of neural response to positive and negative monetary reinforcement. Journal of Neuroscience Methods, 209, 40-49. doi: 10.1016/ j.jneumeth.2012.05.015

Bruin, K., Wijers, A., \& van Staveren, A. S. (2001). Response priming in a go/nogo task: Do we have to explain the go/nogo N2 effect in terms of response activation instead of inhibition? Clinical Neurophysiology, 112, 1660-1671. doi: 10.1016/S1388-2457(01)00601-0

Bruin, K. J., \& Wijers, A. A. (2002). Inhibition, response mode, and stimulus probability: A comparative event-related potential study. Clinical Neurophysiology, 113, 1172-1182. doi: 10.1016/S1388-2457(02) 00141-4

Carter, R. M., MacInnes, J. J., Huettel, S. A., \& Adcock, R. A. (2009). Activation in the VTA and nucleus accumbens increases in anticipation of both gains and losses. Frontiers in Behavioral Neuroscience, 3, 21. doi: 10.3389/neuro.08.021.2009

Cavanagh, J. F., Eisenberg, I., Guitart-Masip, M., Huys, Q., \& Frank, M. J. (2013). Frontal theta overrides Pavlovian learning biases. Journal of Neuroscience, 33, 8541-8548. doi: 10.1523/JNEUROSCI.575412.2013

Chiu, Y.-C., Cools, R., \& Aron, A. R. (2014). Opposing effects of appetitive and aversive cues on go/no-go behavior and motor excitability. Journal of Cognitive Neuroscience, 26, 1851-1860. doi: 10.1162/ jocn_a 00585

Chowdhury, R., Guitart-Masip, M., Lambert, C., Dolan, R. J., \& Düzel, E. (2013). Structural integrity of the substantia nigra and subthalamic nucleus predicts flexibility of instrumental learning in older-age individuals. Neurobiology of Aging, 34, 2261-2270. doi: 10.1016/ j.neurobiolaging.2013.03.030

Cohen, M. X., Cavanagh, J. F., \& Slagter, H. A. (2011). Event-related potential activity in the basal ganglia differentiates rewards from nonrewards: Temporospatial principal components analysis and source localization of the feedback negativity: commentary. Human Brain Mapping, 32, 2270-2271. doi: 10.1002/hbm.21358

Connor, W. H., \& Lang, P. J. (1969). Cortical slow-wave and cardiac rate responses in stimulus orientation and reaction time conditions. Journal of Experimental Psychology, 82, 310-320.

Cui, R. Q., Egkher, A., Huter, D., Lang, W., Lindinger, G., \& Deecke, L. (2000). High resolution spatiotemporal analysis of the contingent negative variation in simple or complex motor tasks and a non-motor task. Clinical Neurophysiology, 111, 1847-1859. doi: 10.1016/S13882457(00)00388-6

Delorme, A., \& Makeig, S. (2004). EEGLAB: An open source toolbox for analysis of single-trial EEG dynamics including independent component analysis. Journal of Neuroscience Methods, 134, 9-21. doi: 10.1016/j.jneumeth.2003.10.009

Eimer, M. (1993). Effects of attention and stimulus probability on ERPs in a go/nogo task. Biological Psychology, 35, 123-138. doi: 10.1016/ 0301-0511(93)90009-W

Engelmann, J. B., Damaraju, E., Padmala, S., \& Pessoa, L. (2009). Combined effects of attention and motivation on visual task performance: Transient and sustained motivational effects. Frontiers in Human Neuroscience, 3, 4. doi: 10.3389/neuro.09.004.2009

Etzel, J. A., Cole, M. W., Zacks, J. M., Kay, K. N., \& Braver, T. S. (2015). Reward motivation enhances task coding in frontoparietal cortex. Cerebral Cortex, 1-13. doi: 10.1093/cercor/bhu327

Falkenstein, M., Hoormann, J., \& Hohnsbein, J. (1999). ERP components in go/nogo tasks and their relation to inhibition. Acta Psychologica, 101, 267-291. doi: 10.1016/S0001-6918(99)00008-6
Falkenstein, M., Hoormann, J., Hohnsbein, J., \& Kleinsorge, T. (2003). Short-term mobilization of processing resources is revealed in the event-related potential. Psychophysiology, 40, 914-923. doi: 10.1111/ 1469-8986.00109

Fan, J., Kolster, R., Ghajar, J., Suh, M., Knight, R. T., Sarkar, R., \& McCandliss, B. D. (2007). Response anticipation and response conflict: An event-related potential and functional magnetic resonance imaging study. Journal of Neuroscience, 27, 2272-2282. doi: 10.1523/JNEUROSCI.3470-06.2007

Filipović, S. R., Jahanshahi, M., \& Rothwell, J. C. (2001). Uncoupling of contingent negative variation and alpha band event-related desynchronization in a go/no-go task. Clinical Neurophysiology, 112, 1307-1315. doi: 10.1016/S1388-2457(01)00558-2

Frank, M. J., Moustafa, A. A., Haughey, H. M., Curran, T., \& Hutchison, K. E. (2007). Genetic triple dissociation reveals multiple roles for dopamine in reinforcement learning. Proceedings of the National Academy of Sciences of the United States of America, 104, 16311-16316. doi: 10.1073/pnas.0706111104

Frank, M. J., Seeberger, L. C., \& O'Reilly, R. C. (2004). By carrot or by stick: Cognitive reinforcement learning in parkinsonism. Science, 306, 1940-1943. doi: 10.1126/science.1102941

Freeman, S. M., Alvernaz, D., Tonnesen, A., Linderman, D., \& Aron, A. R. (2015). Suppressing a motivationally-triggered action tendency engages a response control mechanism that prevents future provocation. Neuropsychologia, 68, 218-231. doi: 10.1016/j.neuropsychologia. 2015.01.016

Funderud, I., Lindgren, M., Løvstad, M., Endestad, T., Voytek, B., Knight, R. T., \& Solbakk, A.-K. (2012). Differential go/nogo activity in both contingent negative variation and spectral power. PloS ONE, 7, e48504. doi: 10.1371/journal.pone.0048504

Gajewski, P. D., \& Falkenstein, M. (2013). Effects of task complexity on ERP components in go/nogo tasks. International Journal of Psychophysiology, 87, 273-278. doi: 10.1016/j.ijpsycho.2012.08.007

Gerschlager, W., Alesch, F., Cunnington, R., Deecke, L., Dirnberger, G., Endl, W., ... Lang, W. (1999). Bilateral subthalamic nucleus stimulation improves frontal cortex function in Parkinson's disease: An electrophysiological study of the contingent negative variation. Brain, 122, 2365-2373. doi: 10.1093/brain/122.12.2365

Goldstein, R. Z., Cottone, L. A., Jia, Z., Maloney, T., Volkow, N. D., \& Squires, N. K. (2006). The effect of graded monetary reward on cognitive event-related potentials and behavior in young healthy adults. International Journal of Psychophysiology, 62, 272-279. doi: 10.1016/ j.ijpsycho.2006.05.006

Goldstein, R. Z., Parvaz, M. A., Maloney, T., Alia-Klein, N., Woicik, P. A., Telang, F., ... Volkow, N. D. (2008). Compromised sensitivity to monetary reward in current cocaine users: An ERP study. Psychophysiology, 45, 705-713. doi: 10.1111/j.1469-8986.2008.00670.x

Gómez, C. M., Flores, A., \& Ledesma, A. (2007). Fronto-parietal networks activation during the contingent negative variation period. Brain Research Bulletin, 73, 40-47. doi: 10.1016/j.brainresbull.2007.01.015

Gray, J. A., \& McNaughton, N. (2000). The neuropsychology of anxiety: An inquiry into the function of the septohippocampal system. Oxford, UK: Oxford University Press.

Grent-'t-Jong, T., \& Woldorff, M. G. (2007). Timing and sequence of brain activity in top-down control of visual-spatial attention. PLoS Biology, 5, e12. doi: 10.1371/journal.pbio.0050012

Guitart-Masip, M., Chowdhury, R., Sharot, T., Dayan, P., Duzel, E., \& Dolan, R. J. (2012). Action controls dopaminergic enhancement of reward representations. Proceedings of the National Academy of Sciences of the United States of America, 109, 7511-7516. doi: 10.1073/ pnas. 1202229109

Guitart-Masip, M., Economides, M., Huys, Q. J. M., Frank, M. J., Chowdhury, R., Duzel, E., ... Dolan, R. J. (2014). Differential, but not opponent, effects of L-DOPA and citalopram on action learning with reward and punishment. Psychopharmacology, 231, 955-966. doi: 10.1007/s00213-013-3313-4

Guitart-Masip, M., Fuentemilla, L., Bach, D. R., Huys, Q. J. M., Dayan, P., Dolan, R. J., \& Duzel, E. (2011). Action dominates valence in anticipatory representations in the human striatum and dopaminergic midbrain. Journal of Neuroscience, 31, 7867-7875. doi: 10.1523/JNEUROSCI.6376-10.2011

Guitart-Masip, M., Huys, Q. J. M., Fuentemilla, L., Dayan, P., Duzel, E., \& Dolan, R. J. (2012). Go and no-go learning in reward and punishment: Interactions between affect and effect. NeuroImage, 62, 154 166. doi: 10.1016/j.neuroimage.2012.04.024 
Hajcak, G., Holroyd, C. B., Moser, J. S., \& Simons, R. F. (2005). Brain potentials associated with expected and unexpected good and bad outcomes. Psychophysiology, 42(2), 161-170. doi:10.1111/j.1469-8986. 2005.00278.x

Hajcak, G., Moser, J. S., Holroyd, C. B., \& Simons, R. F. (2007). It's worse than you thought: The feedback negativity and violations of reward prediction in gambling tasks. Psychophysiology, 44, 905-912. doi: 10.1111/j.1469-8986.2007.00567.x

Hughes, G., Mathan, S., \& Yeung, N. (2012). EEG indices of reward motivation and target detectability in a rapid visual detection task. NeuroImage, 64, 590-600. doi: 10.1016/j.neuroimage.2012.09.003

Ivanov, I., Liu, X., Clerkin, S., Schulz, K., Friston, K., Newcorn, J. H., \& Fan, J. (2012). Effects of motivation on reward and attentional networks: An fMRI study. Brain and Behavior, 2, 741-753. doi: 10.1002/ brb3.80

Jonkman, L. M., Lansbergen, M., \& Stauder, J. E. (2003). Developmental differences in behavioral and event-related brain responses associated with response preparation and inhibition in a go/nogo task. Psychophysiology, 40, 752-761. doi: 10.1111/1469-8986.00075

Kohls, G., Peltzer, J., Schulte-Rüther, M., Kamp-Becker, I., Remschmidt, H., Herpertz-Dahlmann, B., \& Konrad, K. (2011). Atypical brain responses to reward cues in autism as revealed by event-related potentials. Journal of Autism and Developmental Disorders, 41, 1523-1533. doi: 10.1007/s 10803-011-1177-1

Kok, A. (1986). Effects of degradation of visual stimulation on components of the event-related potential (ERP) in go/nogo reaction tasks. Biological Psychology, 23, 21-38. doi: 10.1016/0301-0511(86)90087-6

Krebs, R. M., Boehler, C. N., Appelbaum, L. G., \& Woldorff, M. G. (2013). Reward associations reduce behavioral interference by changing the temporal dynamics of conflict processing. PloS ONE, 8, e53894. doi: 10.1371/journal.pone.0053894

Krebs, R. M., Boehler, C. N., Roberts, K. C., Song, A. W., \& Woldorff, M. G. (2012). The involvement of the dopaminergic midbrain and cortico-striatal-thalamic circuits in the integration of reward prospect and attentional task demands. Cerebral Cortex, 22, 607-615. doi: 10.1093/cercor/bhr134

Kurniawan, I. T., Guitart-Masip, M., Dayan, P., \& Dolan, R. J. (2013). Effort and valuation in the brain: The effects of anticipation and execution. Journal of Neuroscience, 33, 6160-6169. doi: 10.1523/JNEUROSCI.4777-12.2013

Kurniawan, I. T., Guitart-Masip, M., \& Dolan, R. J. (2011). Dopamine and effort-based decision making. Frontiers in Neuroscience, 5, 81. doi: $10.3389 /$ fnins.2011.00081

Linssen, A. M. W., Vuurman, E. F. P. M., Sambeth, A., Nave, S., Spooren, W., Vargas, G., ... Riedel, W. J. (2011). Contingent negative variation as a dopaminergic biomarker: Evidence from dose-related effects of methylphenidate. Psychopharmacology, 218, 533-542. doi: 10.1007/ s00213-011-2345-X

Liu, T., Xiao, T., Shi, J., \& Zhao, D. (2011). Response preparation and cognitive control of highly intelligent children: A go-nogo event-related potential study. Neuroscience, 180, 122-128. doi: 10.1016/j.neuro science.2011.02.022

Locke, H. S., \& Braver, T. S. (2008). Motivational influences on cognitive control: Behavior, brain activation, and individual differences. Cognitive, Affective, \& Behavioral Neuroscience, 8, 99-112. doi: 10.3758/ CABN.8.1.99

Lopez-Calderon, J., \& Luck, S. J. (2014). ERPLAB: An open-source toolbox for the analysis of event-related potentials. Frontiers in Human Neuroscience, 8, 213. doi: 10.3389/fnhum.2014.00213

Lorist, M. M., Klein, M., Nieuwenhuis, S., De Jong, R., Mulder, G., \& Meijman, T. F. (2000). Mental fatigue and task control: Planning and preparation. Psychophysiology, 37, 614-625. doi: 10.1111/1469-8986. 3750614

Ma, Q., Meng, L., Wang, L., \& Shen, Q. (2014). I endeavor to make it: Effort increases valuation of subsequent monetary reward. Behavioural Brain Research, 261, 1-7. doi: 10.1016/j.bbr.2013.11.045

McEvoy, L. K., Smith, M. E., \& Gevins, A. (1998). Dynamic cortical networks of verbal and spatial working memory: Effects of memory load and task practice. Cerebral Cortex, 8, 563-574. doi: 10.1093/cercor/ 8.7.563

Nieoullon, A. (2002). Dopamine and the regulation of cognition and attention. Progress in Neurobiology, 67, 53-83. doi: 10.1016/S03010082(02)00011-4
Nieuwenhuis, S., Aston-Jones, G., \& Cohen, J. D. (2005). Decision making, the P3, and the locus coeruleus-norepinephrine system. Psychological Bulletin, 131, 510-532. doi: 10.1037/0033-2909.131.4.510

Oishi, M., Mochizuki, Y., Du, C., \& Takasu, T. (1995). Contingent negative variation and movement-related cortical potentials in parkinsonism. Electroencephalography and Clinical Neurophysiology, 95, 346-349. doi: 10.1016/0013-4694(95)00084-C

Padmala, S., \& Pessoa, L. (2011). Reward reduces conflict by enhancing attentional control and biasing visual cortical processing. Journal of Neuroscience, 23, 3419-3432. doi: 10.1162/jocn

Paschke, L. M., Walter, H., Steimke, R., Ludwig, V. U., Gaschler, R., Schubert, T., \& Stelzel, C. (2015). Motivation by potential gains and losses affects control processes via different mechanisms in the attentional network. NeuroImage, 111, 549-561. doi: 10.1016/ j.neuroimage.2015.02.047

Pfabigan, D. M., Seidel, E.-M., Sladky, R., Hahn, A., Paul, K., Grahl, A., ... Lamm, C. (2014). P300 amplitude variation is related to ventral striatum BOLD response during gain and loss anticipation: An EEG and fMRI experiment. NeuroImage, 96, 12-21. doi: 10.1016/ j.neuroimage.2014.03.077

Pfefferbaum, A., \& Ford, J. M. (1988). ERPs to stimuli requiring response production and inhibition: Effects of age, probability and visual noise. Electroencephalography and Clinical Neurophysiology, 71, 55-63. doi: 10.1016/0168-5597(88)90019-6

Pfefferbaum, A., Ford, J. M., Weller, B. J., \& Kopell, B. S. (1985). ERPs to response production and inhibition. Electroencephalography and Clinical Neurophysiology, 60, 423-434. doi: 10.1016/0013-4694(85) 91017-X

Pierson, A., Ragot, R., Ripoche, A., \& Lesevre, N. (1987). Electrophysiological changes elicited by auditory stimuli given a positive or negative value: A study comparing anhedonic with hedonic subjects. International Journal of Psychophysiology, 5, 107-123. Retrieved from http:// www.ncbi.nlm.nih.gov/pubmed/3610727

Plichta, M. M., Wolf, I., Hohmann, S., Baumeister, S., Boecker, R., Schwarz, A. J., ... Brandeis, D. (2013). Simultaneous EEG and fMRI reveals a causally connected subcortical-cortical network during reward anticipation. Journal of Neuroscience, 33, 14526-14533. doi: 10.1523/ JNEUROSCI.0631-13.2013

Potts, G. F. (2011). Impact of reward and punishment motivation on behavior monitoring as indexed by the error-related negativity. International Journal of Psychophysiology, 81, 324-331. doi: 10.1016/j.ijpsycho. 2011.07.020

Randall, W. M., \& Smith, J. L. (2011). Conflict and inhibition in the cuedgo/nogo task. Clinical Neurophysiology, 122, 2400-2407. doi: 10.1016/ j.clinph.2011.05.012

Richter, A., Guitart-Masip, M., Barman, A., Libeau, C., Behnisch, G., Czerney, S., ... Schott, B. H. (2014). Valenced action/inhibition learning in humans is modulated by a genetic variant linked to dopamine D2 receptor expression. Frontiers in Systems Neuroscience, 8, 140. doi: 10.3389/fnsys. 2014.00140

Rosahl, S. K., \& Knight, R. T. (1995). Role of prefrontal cortex in generation of the contingent negative variation. Cerebral Cortex, 5, 123-134. doi: 10.1093/cercor/5.2.123

Rösler, F., Heil, M., \& Röder, B. (1997). Slow negative brain potentials as reflections of specific modular resources of cognition. Biological Psychology, 45, 109-141. doi: 10.1016/S0301-0511(96)05225-8

Salamone, J. D., \& Correa, M. (2012). The mysterious motivational functions of mesolimbic dopamine. Neuron, 76, 470-485. doi: 10.1016/ j.neuron.2012.10.021

Salamone, J. D., Correa, M., Farrar, A., \& Mingote, S. M. (2007). Effortrelated functions of nucleus accumbens dopamine and associated forebrain circuits. Psychopharmacology, 191, 461-482. doi: 10.1007/ s00213-006-0668-9

Salamone, J. D., Correa, M., Mingote, S. M., \& Weber, S. M. (2005). Beyond the reward hypothesis: Alternative functions of nucleus accumbens dopamine. Current Opinion in Pharmacology, 5, 34-41. doi: 10.1016/j.coph.2004.09.004

San Martín, R. (2012). Event-related potential studies of outcome processing and feedback-guided learning. Frontiers in Human Neuroscience, 6, 304. doi: 10.3389/fnhum.2012.00304

Sato, A., Yasuda, A., Ohira, H., Miyawaki, K., Nishikawa, M., Kumano, H., \& Kuboki, T. (2005). Effects of value and reward magnitude on feedback negativity and P300. NeuroReport, 16, 407-411. doi: 10.1097/00001756-200503150-00020 
Schevernels, H., Krebs, R. M., Santens, P., Woldorff, M. G., \& Boehler, C. N. (2014). Task preparation processes related to reward prediction precede those related to task-difficulty expectation. NeuroImage, 84 , 639-647. doi: 10.1016/j.neuroimage.2013.09.039

Schmidt, L., Lebreton, M., Cléry-Melin, M.-L., Daunizeau, J., \& Pessiglione, M. (2012). Neural mechanisms underlying motivation of mental versus physical effort. PLoS Biology, 10, e1001266. doi: 10.1371/journal.pbio.1001266

Smith, J. L., Johnstone, S. J., \& Barry, R. J. (2007). Response priming in the go/nogo task: The N2 reflects neither inhibition nor conflict. Clinical Neurophysiology, 118, 343-355. doi: 10.1016/j.clinph.2006.09.027

Smith, J. L., Johnstone, S. J., \& Barry, R. J. (2008). Movement-related potentials in the go/nogo task: The $\mathrm{P} 3$ reflects both cognitive and motor inhibition. Clinical Neurophysiology, 119, 704-714. doi: 10.1016/ j.clinph.2007.11.042

Sobotka, S. S., Davidson, R. J., \& Senulis, J. A. (1992). Anterior brain electrical asymmetries in response to reward and punishment. Electroencephalography and Clinical Neurophysiology, 83, 236-247. doi: 10.1016/0013-4694(92)90117-Z

Tecce, J. J. (1972). Contingent negative variation (CNV) and psychological processes in man. Psychological Bulletin, 77, 73-108. doi: 10.1037/ h0032177

Tekok-Kilic, A., Shucard, J. L., \& Shucard, D. W. (2001). Stimulus modality and go/nogo effects on P3 during parallel visual and auditory continuous performance tasks. Psychophysiology, 38, 578-589. doi: 10.1017/ S0048577201991279

van Boxtel, G. J., \& Brunia, C. H. (1994). Motor and non-motor aspects of slow brain potentials. Biological Psychology, 38, 37-51. doi: 10.1016/ 0301-0511(94)90048-5

van Boxtel, G. J., van der Molen, M. W., Jennings, J. R., \& Brunia, C. H. (2001). A psychophysiological analysis of inhibitory motor control in the stop-signal paradigm. Biological Psychology, 58, 229-262. doi: 10.1016/S0301-0511(01)00117-X

van den Berg, B., Krebs, R. M., Lorist, M. M., \& Woldorff, M. G. (2014). Utilization of reward-prospect enhances preparatory attention and reduces stimulus conflict. Cognitive, Affective \& Behavioral Neuroscience, 14, 561-577. doi: 10.3758/s13415-014-0281-z

Vassena, E., Silvetti, M., Boehler, C. N., Achten, E., Fias, W., \& Verguts, T. (2014). Overlapping neural systems represent cognitive effort and reward anticipation. PloS ONE, 9, e91008. doi: 10.1371/ journal.pone.0091008

Vogel, E. K., \& Luck, S. J. (2000). The visual N1 component as an index of a discrimination process. Psychophysiology, 37, 190-203. Retrieved from http://www.ncbi.nlm.nih.gov/pubmed/10731769

Vuillier, L., Whitebread, D., \& Szucs, D. (2015). ERP evidence of cognitive strategy change in motivational conditions with varying level of difficulty. Neuropsychologia, 70, 126-133. doi: 10.1016/j.neuropsychologia. 2015.02.025

Walter, W. G., Cooper, R., Aldridge, V. J., McCallum, W. C., \& Winter, A. L. (1964). Contingent negative variation: An electric sign of sensorimotor association and expectancy in the human brain. Nature, 203, 380-384. doi: 10.1038/203380a0

Wild-Wall, N., Hohnsbein, J., \& Falkenstein, M. (2007). Effects of ageing on cognitive task preparation as reflected by event-related potentials. Clinical Neurophysiology, 118, 558-569. doi: 10.1016/j.clinph. 2006.09.005

Wu, Y., \& Zhou, X. (2009). The P300 and reward valence, magnitude, and expectancy in outcome evaluation. Brain Research, 1286, 114-122. doi: 10.1016/j.brainres.2009.06.032

Yeung, N., Holroyd, C. B., \& Cohen, J. D. (2005). ERP correlates of feedback and reward processing in the presence and absence of response choice. Cerebral Cortex, 15, 535-544. doi: 10.1093/cercor/ bhh153

Zhou, Z., Yu, R., \& Zhou, X. (2010). To do or not to do? Action enlarges the FRN and P300 effects in outcome evaluation. Neuropsychologia, 48, 3606-3613. doi: 10.1016/j.neuropsychologia.2010.08.010

(Received April 10, 2015; Accepted August 24, 2015) 